\title{
Phase Structure of Internal Gravity Waves in the Ocean with Shear Flows
}

\author{
V. V. Bulatov ${ }^{1, ~}{ }^{凶}$, Yu. V. Vladimirov ${ }^{1}$, I. Yu. Vladimirov ${ }^{2}$ \\ ${ }^{1}$ Ishlinskiy Institute for Problems in Mechanics, Russian Academy of Sciences, \\ Moscow, Russian Federation \\ ${ }^{2}$ P. P. Shirshov Institute of Oceanology, Russian Academy of Sciences, Moscow, Russian Federation \\ 凶internalwave@mail.ru
}

Purpose. The description of the internal gravity waves dynamics in the ocean with background fields of shear currents is a very difficult problem even in the linear approximation. The mathematical problem describing wave dynamics is reduced to the analysis of a system of partial differential equations; and while taking into account the vertical and horizontal inhomogeneity, this system of equations does not allow separation of the variables. Application of various approximations makes it possible to construct analytical solutions for the model distributions of buoyancy frequency and background shear ocean currents. The work is aimed at studying dynamics of internal gravity waves in the ocean with the arbitrary and model distributions of density and background shear currents.

Methods and Results. The paper represents the numerical and analytical solutions describing the main phase characteristics of the internal gravity wave fields in the stratified ocean of finite depth, both for arbitrary and model distributions of the buoyancy frequency and the background shear currents. The currents are considered to be stationary and horizontally homogeneous on the assumption that the scale of the currents' horizontal and temporal variability is much larger than the characteristic lengths and periods of internal gravity waves. Having been used, the Fourier method permitted to obtain integral representations of the solutions under the Miles - Howard stability condition is fulfilled. To solve the vertical spectral problem, proposed is the algorithm for calculating the main dispersion dependences that determine the phase characteristics of the generated wave fields. The calculations for one real distribution of buoyancy frequency and shear flow profile are represented. Transformation of the dispersion surfaces and phase structures of the internal gravitational waves' fields is studied depending on the generation parameters. To solve the problem analytically, constant distribution of the buoyancy frequency and linear dependences of the background shear current on depth were used. For the model distribution of the buoyancy and shear flow frequencies, the explicit analytical expressions describing the solutions of the vertical spectral problem were derived. The numerical and asymptotic solutions for the characteristic oceanic parameters were compared.

Conclusions. The obtained results show that the asymptotic constructions using the model dependences of the buoyancy frequency and the background shear velocities' distribution, describe the numerical solutions of the vertical spectral problem to a good degree of accuracy. The model representations, having been applied for hydrological parameters, make it possible to describe qualitatively correctly the main characteristics of internal gravity waves in the ocean with the arbitrary background shear currents.

Keywords: stratified medium, internal gravity waves, buoyancy frequency, shear flows, vertical spectral problem, dispersion relations, phase patterns

Acknowledgments: the work was done on the following topics of the state task: V.V. Bulatov, Yu.V. Vladimirov (No. AAAA-A20-120011690131-7), I.Yu. Vladimirov (No. 0128-2021-0002), and at partial financial support of the RFBR project No. 20-01-00111A.

For citation: Bulatov, V.V., Vladimirov, Yu.V. and Vladimirov, I.Yu, 2021. Phase Structure of Internal Gravity Waves in the Ocean with Shear Flows. Physical Oceanography, [e-journal] 28(4), pp. 438-453. doi:10.22449/1573-160X-2021-4-438-453

DOI: $10.22449 / 1573-160 X-2021-4-438-453$

(c) V. V. Bulatov, Yu. V. Vladimirov, I. Yu. Vladimirov, 2021

(C) Physical Oceanography, 2021 
Introduction. In the real ocean internal gravity waves (IGW) propagate against the background of shear ocean currents. Therefore, in connection with the progress in the study of large-scale oceanic wave processes, the study of IGW dynamics and propagation in the ocean, taking into account the presence of currents, is an urgent task [1-3]. In the ocean, the vertical and horizontal dynamics of background shear currents is largely associated with internal waves. In the ocean, such currents can manifest themselves, for example, in the region of the seasonal thermocline and have a noticeable effect on the IGW dynamics [4,5]. As a first approximation, it can be assumed that background currents with a vertical velocity shear are weakly dependent on time and horizontal coordinates. If the scale of changes in the currents horizontally is much larger than the IGW lengths, and the scale of temporal variability is much larger than the IGW periods, then such currents can be considered as stationary and horizontally homogeneous $[1,3]$.

In a general formulation, the description of the IGW dynamics in the ocean with background fields of shear currents is a very difficult problem in the linear approximation yet. In this case, the problem is reduced to the analysis of a system of partial differential equations, and while taking into account the vertical and horizontal inhomogeneity, this system of equations does not allow the separation of variables. Using various approximations, including the WKB method, based on a realistic assumption about the smoothness of variations in the parameters of the oceanic medium in comparison with the IGW lengths, it is possible to construct analytical solutions for the model distributions of the buoyancy and shear currents $[3,5,6]$. Therefore, it is of undoubted interest to study IGW in the ocean with an arbitrary vertical distribution of both the density and the background shear currents observed under sea conditions [4, 7-9]. Thus, it becomes possible to study the amplitude-phase characteristics of the wave fields of real oceanological parameters.

One of the main methods for solving problems of IGW wave dynamics in the ocean with arbitrary background shear currents is the Fourier method, which provides the construction of integral representations of solutions requiring numerical and asymptotic analysis [10-15]. Direct numerical modeling methods are not always effective for studying IGW generation by arbitrary non-local and non-stationary sources of disturbances, especially taking into account the variability of the main hydrological parameters, and require verification and comparison with solutions of model problems $[5,16]$. Therefore, when analyzing the IGW dynamics in the real ocean, various asymptotic and analytical models are useful [3, 6, 14, 17-20].

In order to study the mechanism of the effect of currents on IGW, it is necessary to consider fairly simple model concepts for stratification and shear currents. The synthesis of various analytical and asymptotic results can give an initial qualitative and quantitative understanding of wave oceanic processes [1, 3, 5]. It can also be expected that taking into account the real properties of the parameters of the marine environment will make it possible to study qualitatively new effects of wave generation. Asymptotic solutions require verification of the results obtained with their help; therefore, comparison of numerical and asymptotic results is of particular interest for studying the dynamics of IGW in the ocean with currents $[3,5,16]$.

The aim of this work is to study the dynamics of IGW in the ocean with a shear of the current velocity using numerical and analytical solutions that describe the 
main features of the dispersion dependences and phase characteristics of IGW fields in a stratified ocean of finite depth for arbitrary and model vertical distributions of the buoyancy frequency and background shear currents.

Problem statement, integral forms of solutions. We consider a vertically stratified medium of finite depth $H$. The linearized system of hydrodynamic equations with respect to the unperturbed state has the form [1-3]

$$
\begin{aligned}
& \rho_{0}\left(\frac{D U_{1}}{D t}+\frac{d V}{d z} W\right)+\frac{\partial p}{\partial x}=0, \quad \rho_{0}\left(\frac{D U_{2}}{D t}+\frac{d U}{d z} W\right)+\frac{\partial p}{\partial y}=0, \quad \rho_{0} \frac{D W}{D t}+\frac{\partial p}{\partial z}+\rho g=0, \\
& \frac{\partial U_{1}}{\partial x}+\frac{\partial U_{2}}{\partial y}+\frac{\partial W}{\partial z}=Q, \quad \frac{D \rho}{D t}+W \frac{\partial \rho_{0}}{\partial z}=0, \quad \frac{D}{D t}=\frac{\partial}{\partial t}+V(z) \frac{\partial}{\partial x}+U(z) \frac{\partial}{\partial y},
\end{aligned}
$$

where $V(z), U(z)$ are the components of the background shear current vector at $z$ horizon; $U_{1}, U_{2}, W$ are the components of perturbed velocity; $p, \rho$ are pressure and density disturbances; $\rho_{0}(z)$ is unperturbed density of the marine environment; $Q(t, x, y, z)$ is the density distribution of mass sources. Cross differentiation from this system of equations using the Boussinesq approximation can be used to obtain one equation for small perturbations of the vertical velocity component $[1-3,5,6,19]$ :

$$
\begin{gathered}
L W=\frac{D}{D t}\left(\frac{\partial}{\partial z}\left(\frac{D Q}{D t}\right)\right), \\
L \equiv \frac{D^{2}}{D t^{2}}\left(\Delta+\frac{\partial^{2}}{\partial z^{2}}\right)-\frac{D}{D t}\left(\frac{d^{2} U}{d z^{2}} \frac{\partial}{\partial x}+\frac{d^{2} V}{d z^{2}} \frac{\partial}{\partial y}\right)+N^{2}(z) \Delta, \\
\Delta=\frac{\partial^{2}}{\partial x^{2}}+\frac{\partial^{2}}{\partial y^{2}}, \quad N^{2}(z)=-\frac{g}{\rho_{0}(z)} \frac{d \rho_{0}(z)}{d z},
\end{gathered}
$$

where $N^{2}(z)$ is the square of Brunt - Väisälä frequency (buoyancy frequency); $g$ is a gravitational acceleration. The boundary conditions are taken in the form (vertical axis $z$ is directed upwards): $W=0$ at $z=0,-H$. Next, we consider the Green's function of equation (1), i.e the solution of the problem [6, 19]:

$$
\begin{gathered}
L \Gamma\left(t, x, y, z, z^{\prime}\right)=\delta(t) \delta(x) \delta(y) \delta\left(z-z^{\prime}\right), \\
\Gamma=0 \quad \text { at } z=0, \quad-H ; \Gamma \equiv 0 \quad \text { at } t<0,
\end{gathered}
$$

where $z^{\prime}$ is the depth of the point source of disturbances and it is assumed that the point source is turned on at time $t=0$, and at $t<0$ the medium is in a state of rest $[6,19]$. Wave disturbances from an arbitrary non-stationary non-local source of disturbances are determined by the corresponding convolution $[1,6,19]$. In the presence of background shear currents in the ocean, IGW can interact with these currents and exchange energy with them; therefore, natural wave oscillations can be exponentially growing. Further, it is assumed that the Miles - Howard stability condition for the Richardson number is satisfied [3, 10-12]: $\operatorname{Ri}(z)=N^{2}(z)\left(\left(\frac{d V}{d z}\right)^{2}+\left(\frac{d U}{d z}\right)^{2}\right)^{-1 / 2}>1 / 4$. If the Miles - Howard condition is satisfied, then the corresponding spectral problem has no complex eigenvalues 
$[3,14]$. The characteristic values of the Richardson numbers in different areas of the World Ocean in the absence of dynamic instability of background shear currents can be within 2-20 intervals [4, 7-9].

The solution to problem (2) is sought in the form of the Fourier integrals

$$
\Gamma\left(t, x, y, z, z^{\prime}\right)=\frac{1}{4 \pi^{2}} \int_{-\infty}^{\infty} d \mu \int_{-\infty}^{\infty} d v \int_{-\infty}^{\infty} G\left(\omega, \mu, v, z, z^{\prime}\right) \exp (-i(\mu x+v y-\omega t)) d \omega .
$$

Then from the condition $\Gamma \equiv 0$ for $t<0$ it follows that the poles and cuts when integrating with respect to the variable $\omega$ must be bypassed in the upper half-plane $[3,6,19]$. The function $G(\omega, \mu, v, z, z)$ is a solution to the following problem [3, 12, 19]:

$$
\begin{gathered}
\Pi G\left(\omega, \mu, v, z, z^{\prime}\right)=\delta\left(z-z^{\prime}\right), \\
\Pi \equiv(\omega-f(z))^{2} \frac{\partial^{2}}{\partial z^{2}}+k^{2}\left(N^{2}(z)-(\omega-f(z))^{2}\right)+\frac{\partial^{2} f(z)}{\partial z^{2}}(\omega-f) ; \\
G=0 \text { at } z=0,-H ; \quad k^{2}=\mu^{2}+v^{2} ; \quad f(z)=\mu V(z)+v U(z) .
\end{gathered}
$$

Numerical algorithm and calculation results. Spectral problem (3) for arbitrary distributions of functions $N(z), V(z), U(z)$ admits only a numerical solution. In order to solve this problem, you can use a numerical algorithm based on the approximation of the main coefficients (3) by piecewise linear and piecewise constant functions. We will consider $\omega=\omega_{n}(\mu, v)$ a spectral parameter that requires to be determined, $\mu$ and $v$ are independent variables.

The eigenfunction $\varphi(z)$ satisfies the equation

$$
\Pi \varphi(z)=0,
$$

$\varphi=0$ at $z=0,-H$.

Further we introduce the new function $F(z)=\varphi(z) /(\omega-f(z))$ which is determined from

$$
\frac{\partial}{\partial z}\left((\omega-f(z))^{2} \frac{\partial F}{\partial z}\right)+k^{2}\left(N^{2}(z)-(\omega-f(z))^{2}\right) F=0,
$$

$F(z)=0$ at $z=0,-H$.

For numerical solution of the problem (4), the interval of variation $-H<z<0$ is divided into $J$ sections (layers): $I_{j}=\left[z_{j-1}, z_{j}\right], j=1,2, \ldots, J$, where $z_{0}=0, z_{J}=-H$. We approximate the coefficient $\omega-f(z)$ in front of function $\frac{\partial F(z)}{d z}$ by a piecewiselinear continuous function, the coefficient in front of $F(z)$ function - by a piecewiseconstant function, i.e. we will assume that $\omega-f(z)=A_{j}+B_{j}\left(z-z_{j}\right)==D_{j}+B_{j} z$; $N^{2}(z)-(\omega-f(z))^{2}=C_{j}^{2} ; \quad z_{j-1}<z<z_{j}$. Then, in each layer the Euler differential equation is obtained to determine the function $F(z)$.

It is known that the eigenvalues of the spectral problem (4) form two series [3, 6, $12-15,19]$. In the first series $\omega_{n}(\mu, v)$ decreases with increasing $n$ and tends to $f_{+}$at 
$n \rightarrow \infty$ ( $f_{+}$is the maximum $f(z)$ value on the variation interval of variable $\left.z\right)$. In the second series $\omega_{n}(\mu, v)$ increases with increasing $n$ and tends to $f_{-}$at $n \rightarrow \infty$ ( $f_{-}$is the minimum value in the variation interval of variable $z$ ). This behavior feature of the dispersion relations is used to find the eigenvalues. Choosing any initial approximation $\theta$ so that $\theta>f_{+}$, is necessary to integrate equation (4), using the following as initial values: $F(z)=0, \frac{\partial F(z)}{d z}=1$ at $z=0$. Если найденное решение имеет $m$ корней внутри интервала $-H<Z<0$ и $m<n$ If the solution we found has $m$ roots inside $-H<z<0$ interval and $m<n$, then $\theta$ value decreases; if $m>n$, then $\theta$ increases. By choosing $\theta$ value, it is possible to achieve that the solution vanishes at $\mathrm{z}=-H$ and has exactly $n$ roots inside the interval $-H<\mathrm{z}<0$. The resulting value of $\omega$ is an eigenvalue and the corresponding solution $F(z)$ is an eigenfunction. Then the eigenfunction $\varphi(z)$ is determined from the relation $\varphi(z)=F(z)(\omega-f(z))$. The performed testing numerical calculations demonstrated good convergence of the proposed numerical algorithm with an increase in the number of layers $J$.

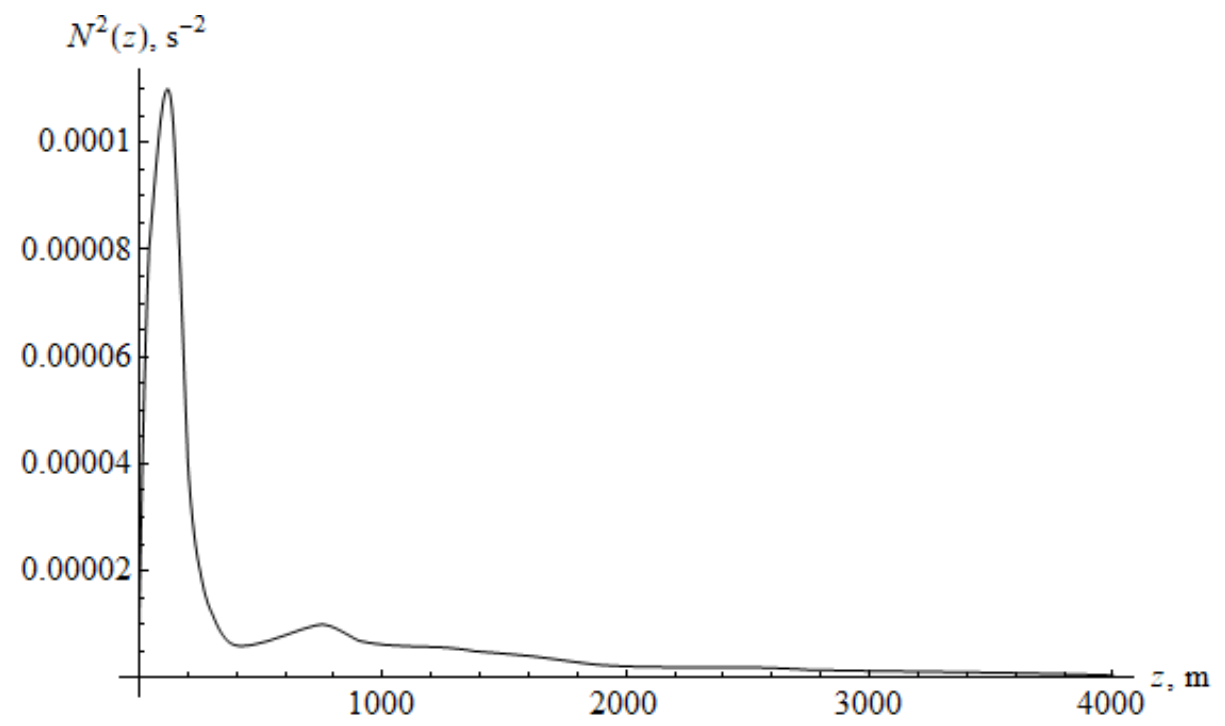

F i g. 1. Buoyancy frequency distribution

For numerical calculations we used typical distributions of the square of the buoyancy frequency $N^{2}(z)$, as well as the components of the shear current $V(z)$ (Fig. 1, 2), which are typical for many regions of the World Ocean, in particular for the water area of the North Atlantic (passages of the East Azores Ridge) [4 , 7-9]. The shear flow component $U(z)$ is assumed to be equal to zero: $U(z)=0$. 


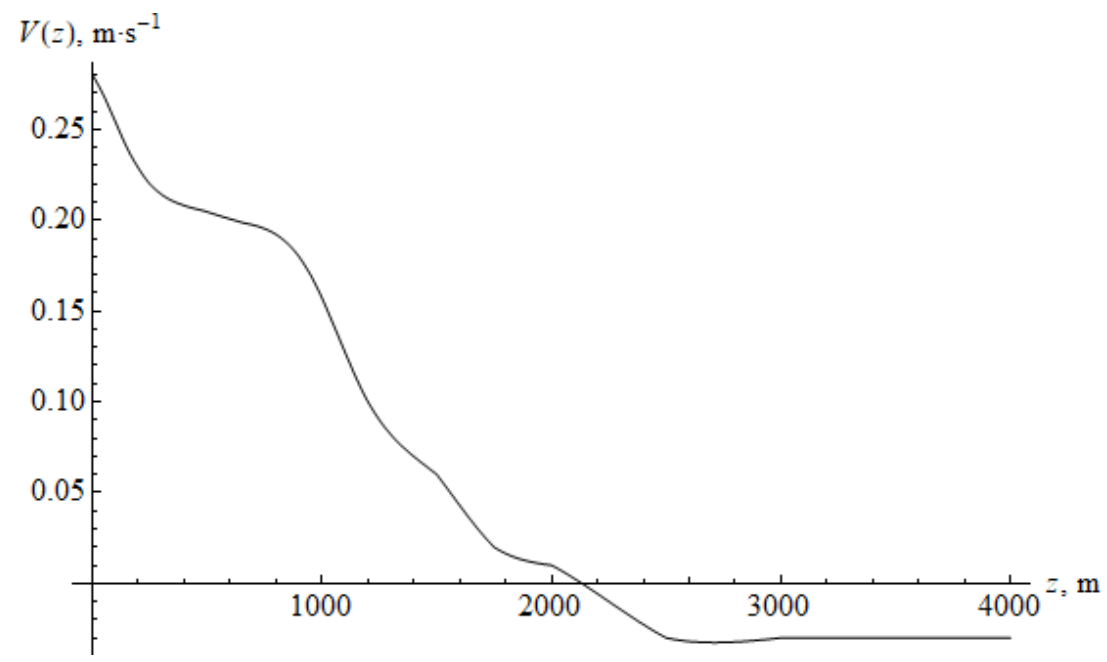

F i g. 2. Shear flow distribution by depth

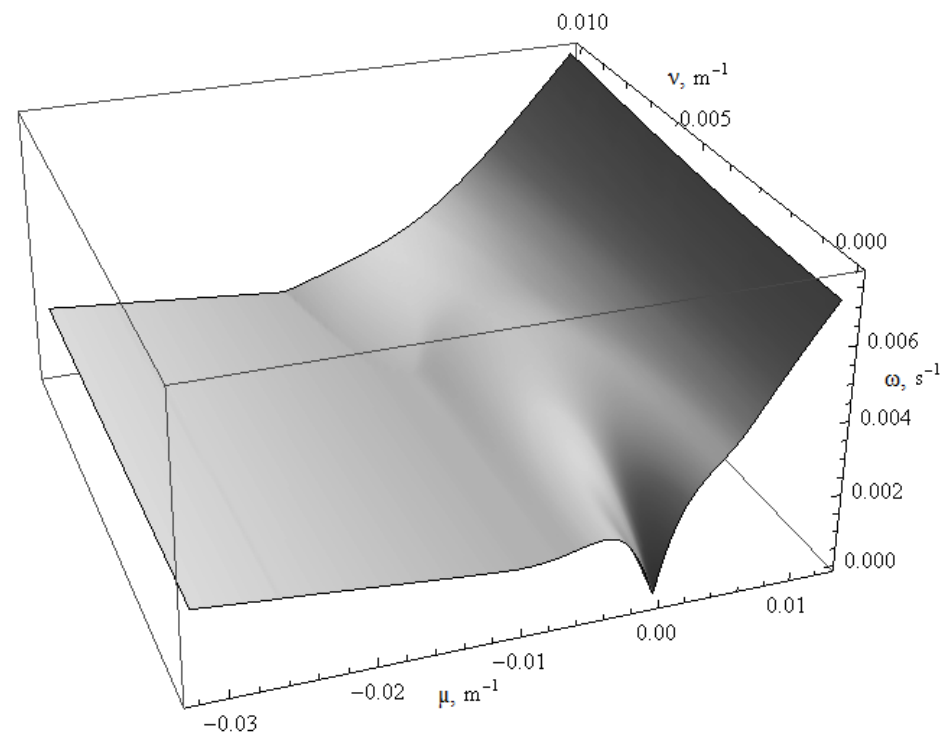

F i g. 3. Dispersion surface

Without loss of generality and for greater clarity of the obtained results, all numerical calculations are given for the second wave mode. In Fig. 3 the results of calculations of the dispersion surface $\omega 2(\mu, v)$ are demonstrated. The structure of local extrema and other singular points in the space of the wave vector components $(v, \mu)$ determines the corresponding features of the phase structures of excited IGW wave fields in Cartesian coordinates $(x, y)$ [21, 22]. As can be seen from the presented numerical results, the dispersion surface has a rather complex spatial structure. Numerically calculated dispersion surfaces can have several local extrema, which corresponds to the generation of various types of wave structures $[17,18,20$, 21]. In Fig. 4 and 5 the results of calculating the dispersion dependences $\mu_{2}(v, \omega)$ for 
different values of $\omega$ frequency are given. In Fig. $4 \omega=0.0013 \mathrm{~s}^{-1}$ (curve 1), $\omega=$ $0.0016 \mathrm{~s}^{-1}$ (curve 2), $\omega=0.00185 \mathrm{~s}^{-1}$ (curve 3); in Fig. $5 \omega=0.00215 \mathrm{~s}^{-1}$ (curve 4), $\omega=0.0038 \mathrm{~s}^{-1}$ (curve 5).

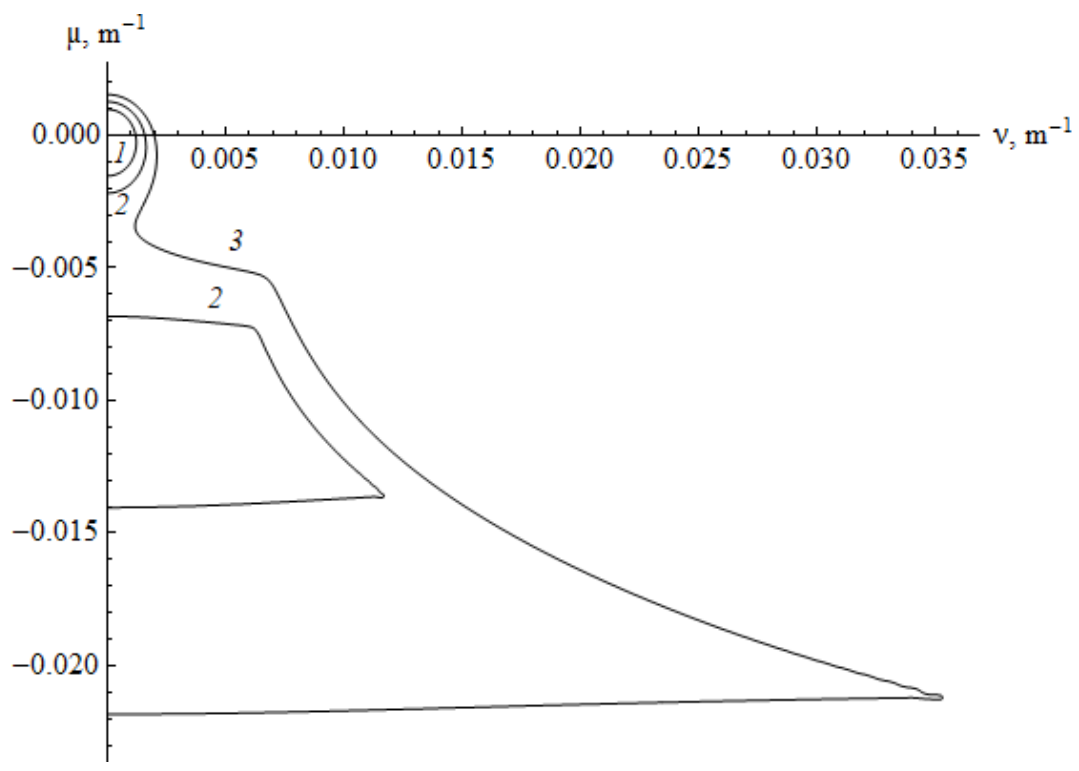

F i g. 4. Dispersion dependences for different values of $\omega$ : line 1 - one closed arc; lines 2 - two closed arcs; line 3 - one closed curve with three inflection points

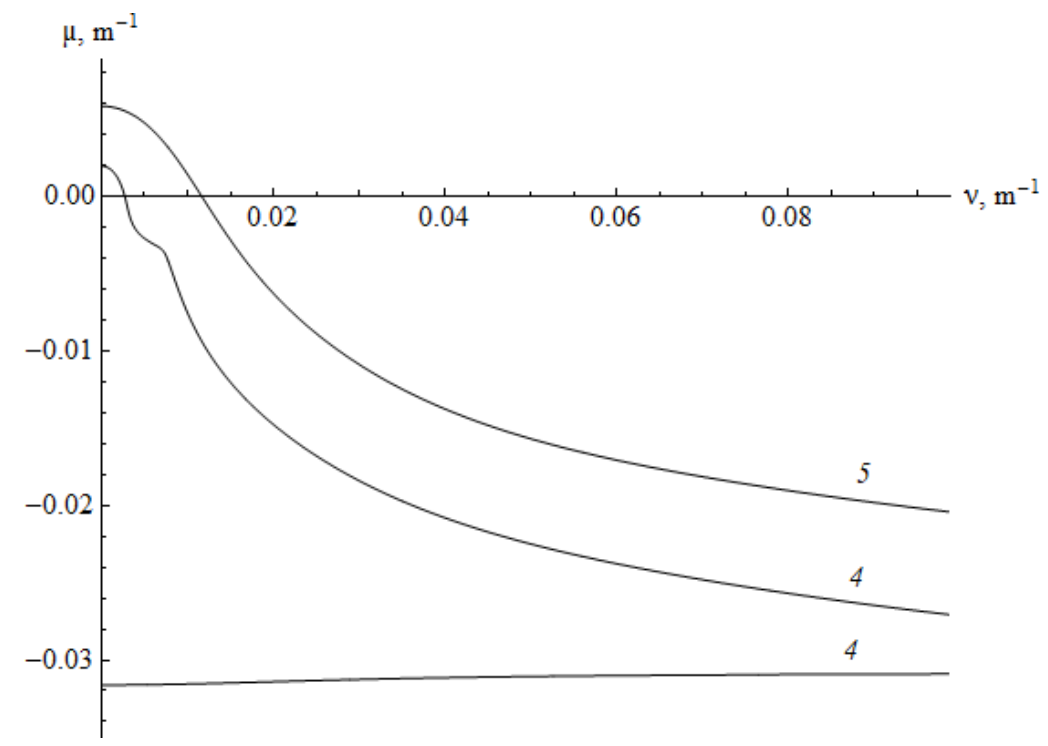

F i g. 5. Dispersion dependences for different values of $\omega$ : lines 4 - two open curves, upper curve 4 with three inflection points; line 5 - upper curve with one inflection point

Further we are to describe the qualitative evolution of the dispersion relations depending on the variation in parameter $\omega$. At small $\omega$ values, the dispersion curve 
has the form of a single closed curve (Fig. 4). As $\omega$ increases and depending on the parameters of the stratified medium (the buoyancy frequency and shear flow), the dispersion curves can consist of several closed curves (Fig. 5). Numerical calculations show that with an increase in the mode number, the number of such closed curves, as a rule, increases. Some of the open curves can join together (Fig. 4, 5) and merge into one curve, which has an ambiguous omega-shaped structure with several inflection points. For sufficiently large $\omega$ values, all dispersion dependences have the form of several open single-valued curves (Fig. 5). The presence of inflection points of dispersion curves determines the locus of points (caustics, wave fronts) where the wave field can change its qualitative behavior [6, 17, 18, 20-22].

These features of the dispersion relations mean that the total wave field of excited IGWs in the ocean with background shear currents is the sum of several types of wave structures. Closed dispersion curves describe the generation of ring (shear) waves, with each branch corresponding to a separate wave system. Simple arcs of dispersion curves describe the generation of wedge-shaped (longitudinal) waves; each arc corresponds to a separate wave system $[17,18,20]$. Numerical calculations for various oceanic distributions of the background shear current components show that the alternating sign of the flow, as a rule, leads to the appearance of closed curves of dispersion relations and, accordingly, to the generation of ring (shear) waves. If the current throughout the ocean depth does not change its sign, then the dispersion curves are a set of only simple arcs and all excited waves are wedge-shaped (longitudinal).

In Fig. 6 and 7 the results of numerical calculations of the IGW phase structure for various $\omega$ frequency values given. The lines of equal phase $\Omega$ of each wave mode are specified parametrically (with the parameter $v$ ) $[17,18]$ :

$$
x(v)=(\omega t-\Omega) /\left(\mu_{n}(v)-v \mu_{n}^{\prime}(v)\right), y(v)=-\mu_{n}^{\prime}(v)(\omega t-\Omega) /\left(\mu_{n}(v)-v \mu_{n}^{\prime}(v)\right) .
$$

The lines of equal phase $\Omega$ in the figures are solid curves, dashed lines are the corresponding wave fronts (caustics). In Fig. 6, the parameter values were as follows: $\omega=0.0026 \mathrm{~s}^{-1}, t=3600 \mathrm{~s}$. The half-opening angle of the wave wedge (external) is $\alpha_{1}=52.44^{\circ}$, the half-opening angle of the inner wedge is $\alpha_{0}=31.46^{\circ}\left(\alpha_{0}\right.$ direction is set by the third dashed ray from the left). The value $\alpha_{0}$ is defined by the equality $\alpha_{0}=-\operatorname{arctg} v_{0}$, where $v_{0}$ is the positive root of the equation $\mu_{2}^{\prime}(v)=\mu_{2}(v) / v$. The half-angle values of the other three wavefronts $\alpha_{k}(k=1,2,3)$ are determined by the local extrema of the function $\mu_{2}^{\prime}(v): \alpha_{k}=-\operatorname{arctg}\left(\mu_{2}^{\prime}\left(v_{k}^{*}\right)\right)$, where $v_{k}^{*}$ are the roots of the equation $\mu^{\prime \prime}{ }_{2}\left(v_{k}^{*}\right)=0$. Waves in the inner wedge (waves of the first type) run to the left (to the origin), waves in the outer wedge (waves of the second type) run to the right (from the origin). Phase along the crests of the first-type waves (from the left to the right): $\Omega=\{4 \pi, 6 \pi, 8 \pi, 10 \pi, 12 \pi, 14 \pi\}$, phase along the crests of the second-type waves (from right to left): $\Omega=\{-2 \pi, 0,2 \pi\}$. 


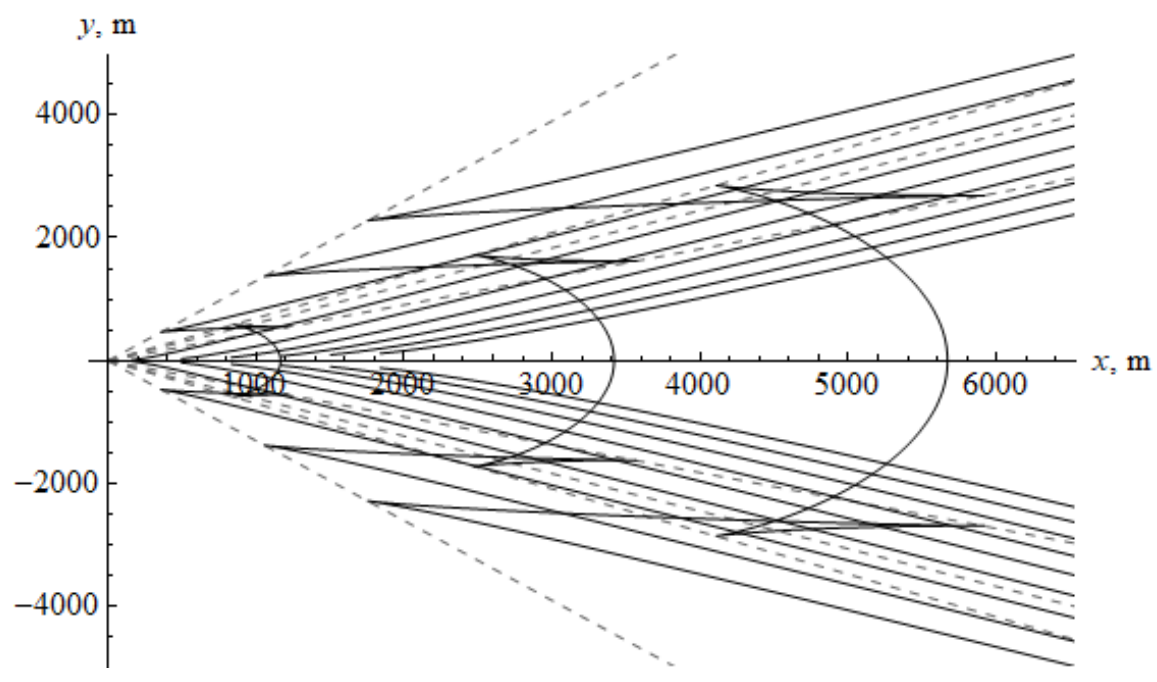

F i g. 6. Equal phase lines for two wave systems

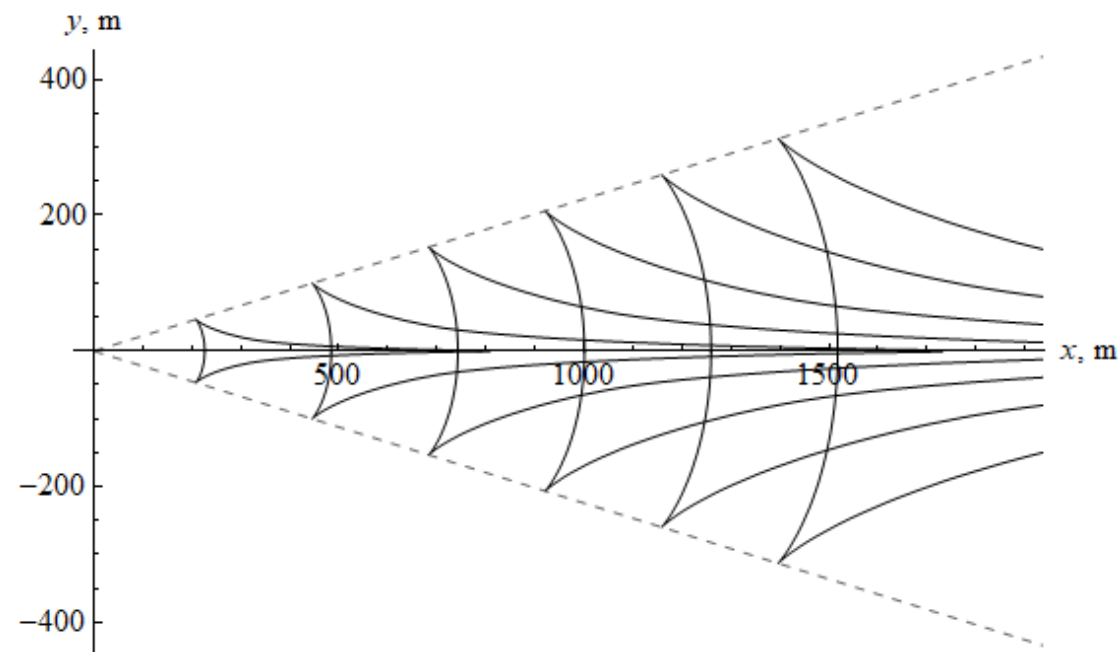

F i g. 7. Equal phase lines for one wave system

At a certain time moment $\tau=\Omega / \omega$, the first-type wave transforms into the second-type wave. The other two pairs of wave fronts form angles $\alpha_{2}=34.79^{\circ}$ and $\alpha_{3}=24.46^{\circ}$ with the $x$ axis, respectively. In Fig. 7, the parameter values are as follows: $\omega=0.0012 \mathrm{~s}^{-1}, t=3600 \mathrm{~s}$. Waves are limited by a wave front with a halfopening angle $\alpha_{1}$, where $\alpha_{1}=\operatorname{arctg}\left(\mu_{2}^{\prime}\left(v^{*}\right)\right), v^{*}$ is the root of the equation $\mu^{\prime \prime}{ }_{2}\left(v^{*}\right)=$ $=0$. The half-opening angle of the wave wedge is $\alpha_{1}=12.63^{\circ}$. The waves inside the wedge run to the right (from the origin), the phase along the wave crests (from right to left): $\Omega=\{4 \pi, 6 \pi, 8 \pi, 10 \pi, 12 \pi\}$.

Thus, numerical calculations for various modes of wave generation demonstrate a wide variety of excited IGWs in the ocean with arbitrary vertical distributions of the buoyancy frequency and background shear currents. For a detailed analysis of 
dispersion relations, it is possible to use model representations of hydrological parameters, which qualitatively correctly describe both the nature and the scale of the spatial variability of shear oceanic currents over the depth.

Asymptotic solutions. Model representations for the buoyancy frequency shear flow provide the construction of asymptotic solutions. The following assumptions are used: the Brunt - Väisälä frequency is constant $(N(z)=N=$ const $)$; background shear flow is one-dimensional $(U(z) \equiv 0) ; V(z)=V_{0}+\left(V_{0}-V_{H}\right) z / H$, $V_{0}=V(0), V_{H}=V(-H)$ is a linear function of depth.

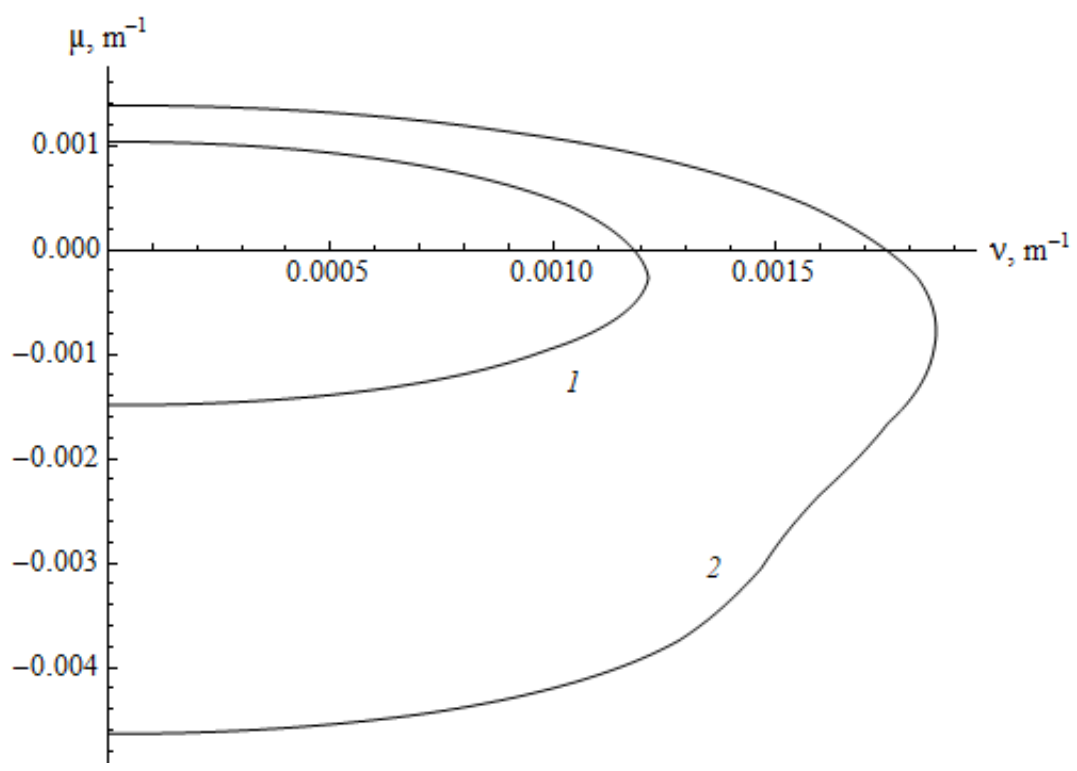

F i g. 8. Dispersion dependences for different values of $\omega$ : line 1 - one closed arc; line 2 - one closed arc

In Fig. 8-10 the results of calculations of the dispersion dependences $\mu_{2}(v, \omega)$ for averaged real conditions and the same values of the frequency $\omega$. In Fig. $8 \omega==$ $0.0013 \mathrm{~s} \mathrm{~s}^{-1}$ (curve 1), $\omega=0.0016 \mathrm{~s}^{-1}$ (curve 2); in Fig. $9 \omega=0.00185 \mathrm{~s}^{-1}$ (curve 3); in Fig. $10 \omega=0.00215 \mathrm{~s}^{-1}$ (curve 4), $\omega=0.0038 \mathrm{~s}^{-1}$ (curve 5). The constant averaged buoyancy frequency is $\frac{1}{\mathrm{H}} \int_{-\mathrm{H}}^{0} \mathrm{~N}(\mathrm{z}) \mathrm{dz}=0.00295 \mathrm{~s}^{-1}, \mathrm{~V}_{0}=0.28 \mathrm{~m} \mathrm{~s}^{-1}$, $\mathrm{V}_{\mathrm{H}}=-0.03 \mathrm{~ms}^{-1}, \mathrm{H}=4000 \mathrm{~m}$. 


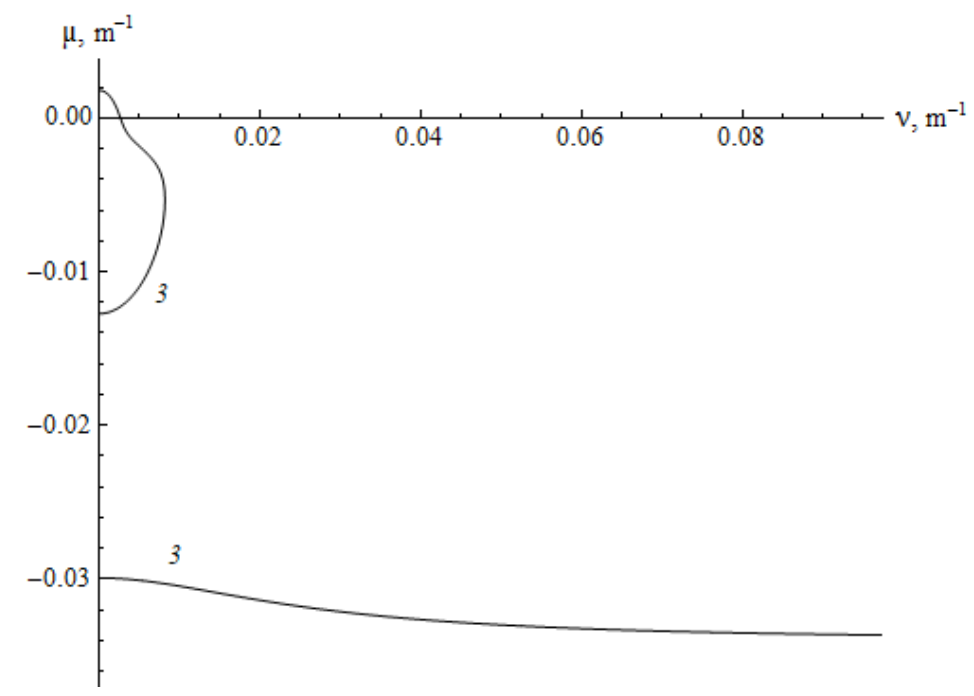

F i g. 9. Dispersion dependences for different values of $\omega$ : lines 3 - one closed arc, one open curve with one inflection point

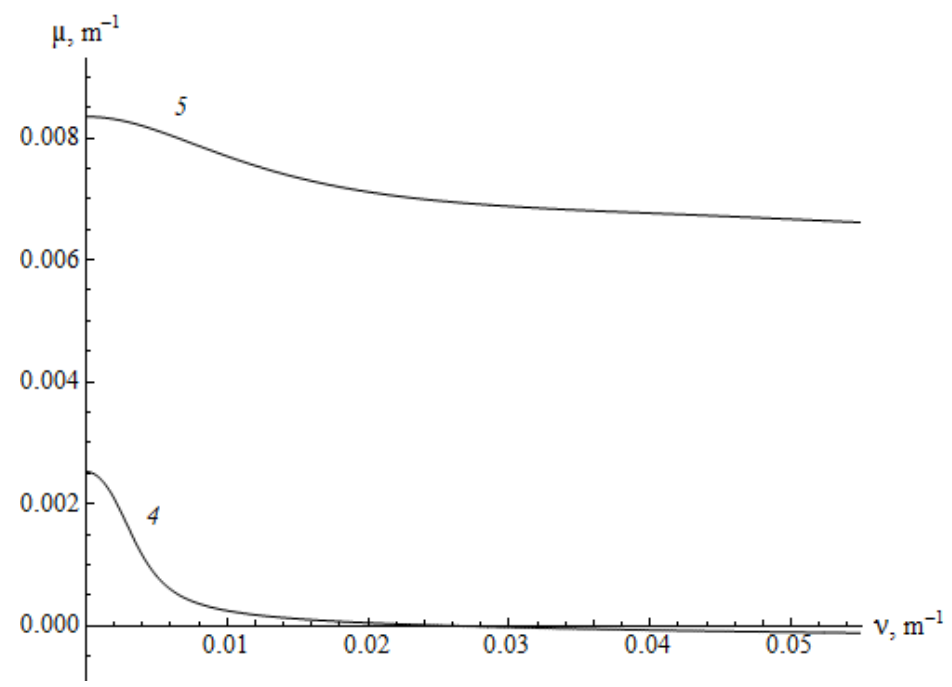

F i g. 10. Dispersion dependences for different values of $\omega$ : line 4 - one open curve with one inflection point; line 5 - upper curve with one inflection point

An analysis of the numerical results shows that the model representations of hydrological parameters make it possible to describe the main qualitative features of the dispersion relations, in particular, to obtain closed dispersion curves for small $\omega$ values describing the generation of ring (shear) waves. At large $\omega$ values the dispersion dependences for both real and model distributions of buoyancy frequency and shear flows have the form of two open curves. Numerical calculations using the real distribution of the buoyancy and shear flow frequencies show that a dispersion pattern (depending on the frequency $\omega$ ), at which there are more than two inflection points, which means that several wave trains contribute to far IGW field, can be observed. A more complex structure of dispersion relations, including the presence 448 
of more than two inflection points, can be investigated only by numerical methods using real oceanological data.

However, using the model representations, problem (4) can be solved analytically. In this case, the dispersion relation has the form $[17,18,20]$

$$
\operatorname{Im}\left(I_{i \lambda}(\beta r(0))_{-i \lambda}(\beta r(-\pi))\right)=0,
$$

where $I_{ \pm i \lambda}$ is a modified Bessel function of imaginary index $i \lambda$; $(z)=(\omega-\mu V(z)) / N ; \quad \lambda=\sqrt{\beta^{2}-1 / 4} ; \beta=k / b \mu b=\left(V_{0}-V_{H}\right) / N H[17,18,20,23]$. The solution of equation (6) $\varphi(z, \mu)$ has the form $[17,18,20]$

$$
\begin{gathered}
\varphi(z, \mu)=i\left(\Psi_{+}(0, \mu) \Psi_{-}(z, \mu)-\Psi_{-}(0, \mu) \Psi_{+}(z, \mu)\right), \\
\Psi_{ \pm}(z, \mu)=\sqrt{2 \beta r(z)} I_{ \pm i \lambda}(\beta r(z)) .
\end{gathered}
$$

Dispersion equation (5) does not have exact analytical solutions; however, one can find their asymptotics for large Richarson numbers. The Richardson number used in this model is $\mathrm{Ri}=767>>1 / 4$. Equation (5) is solved by two families of dispersion curves: $\mu^{\mathrm{n} 1}(v), \mu_{\mathrm{n} 2}(v)$. Firstly we consider the family $\mu_{\mathrm{n} 1}(v)$. All curves of this family are within the interval $\left(\mu_{d}, \mu_{a}\right)$, which is divided by the turning point into two intervals. In the interval $\left(\mu_{\mathrm{c}}, \mu_{\mathrm{a}}\right)$, both functions from (6) oscillate. Replacing each of them with asymptotics, one can obtain $[17,18,20,23]: \beta(\Theta(r(-\pi))-\Theta(r(0)))$ $=\pi n$. The solution to this equation has the form

$$
v_{n}(\mu)= \pm\left(\left(\pi b n /(\Theta(r(-\pi))-\Theta(r(0)))^{2}-1\right) \mu^{2}\right)^{1 / 2} .
$$

It is possible to obtain from equation (7) the inverse dependence $\mu_{n 1}(v)$ using a standard computing system of the "Mathematics" type. In the interval $\left(\mu_{d}, \mu_{c}\right)$, the second function in (6) oscillates, the first no longer oscillates. Therefore, replacing these functions with the corresponding asymptotics [17, 18, 20, 23], we can obtain

$$
|\beta| \Theta(r(-\pi))-\pi / 4+\operatorname{arctg}(\exp (-2|\beta| \Phi(r(0)))) / 2=-\pi n .
$$

In contrast to (5), equation (8) can be easily solved numerically, since the lefthand side (asymptotic approximation of the phase) is a strictly monotonic function. In Fig. 11 the dispersion curves of the second mode $\mu_{21}(v)$, calculated numerically from equation (4) (solid line), and their asymptotic approximations, calculated by formulas (7), (8) (dashed lines) are represented. The second family of dispersion curves $\mu_{\mathrm{n} 2}(v)$ lies in the interval $\left(\mu_{\mathrm{b}}, \mu_{\mathrm{e}}\right)$. In this case, it is necessary to take into account the contribution to the phase of the second function from (8), and as a result, we can obtain the asymptotics of equation (5): $|\beta| \Theta(\mathrm{r}(-\pi))=\pi / 4-\pi \mathrm{n}$. The solution to this equation is described by expression (8), using which the dependence $\mu_{\mathrm{n} 2}(v)$ can be obtained. In Fig. 12 the results of $\varphi_{22}(z)$ calculations - the eigenfunction of the second mode, normalized to its maximum value, is demonstrated. 


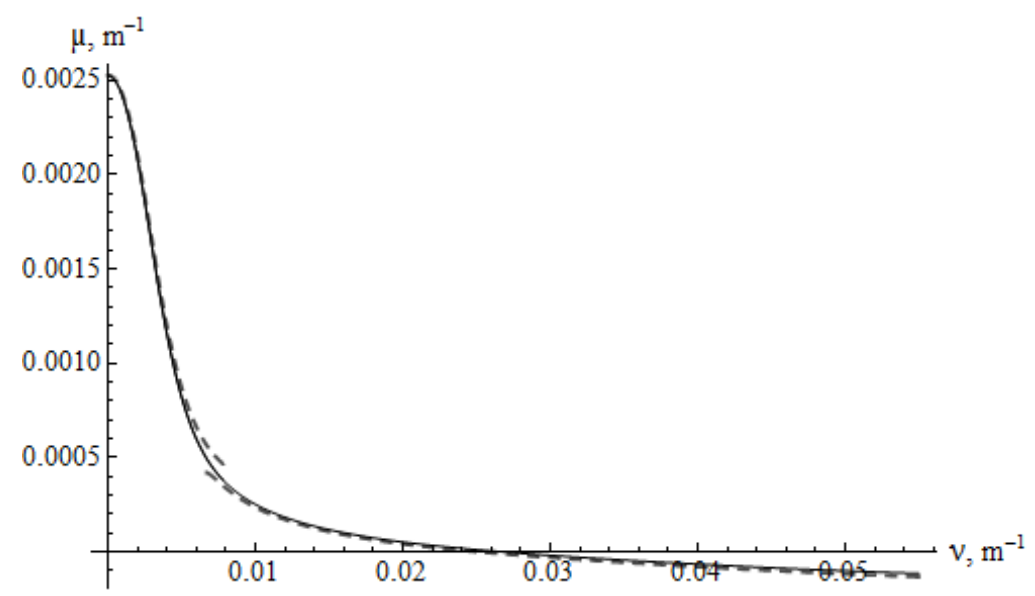

F i g. 11. Dispersion curves of the second mode

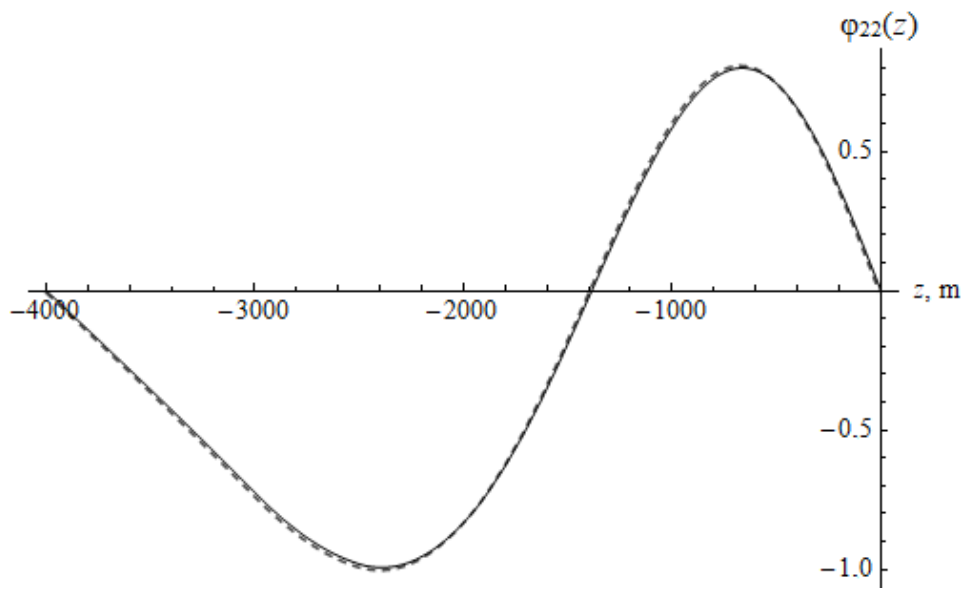

F i g. 12. Eigenfunction of the second mode at $v=0,01 \mathrm{~m}^{-1}, \mu=0,00025 \mathrm{~m}^{-1}, \omega=0,002 \mathrm{~s}^{-1}$. Solid line numerical result (4), dashed line - asymptotics (7)

The obtained results demonstrate a good agreement between the asymptotics and numerical calculations; therefore, the use of model representations for hydrological parameters makes it possible to qualitatively correctly describe the main characteristics of IGW in the ocean with background shear currents. It can be noted, in particular, that with the use of hydrological parameters the flow does not change its sign over the entire depth of the ocean, the dispersion curves consist of completely open branches and therefore only wedge-shaped (longitudinal) waves can be excited $[17,18,20]$.

The analysis of numerical calculations showed that taking into account the real distributions of the main hydrological parameters of the ocean makes it possible to study the whole variety of generated wave systems. A change in the main parameters of wave generation causes a noticeable qualitative rearrangement of the phase patterns of the excited IGW fields, associated with the transformation of dispersion 
dependences. The use of analytical and asymptotic methods makes it possible to determine the main characteristics of IGW in the ocean with background shear currents. Therefore, in order to study IGW in a real ocean, it is necessary to combine both precise numerical methods for studying wave fields and various asymptotic approaches that make it possible to study the main qualitative features of the excited waves. In the general case, the solution of the dispersion equation and the qualitative analysis of the dispersion relations is a significant mathematical difficulty $[3,12-15$, 19].

The obtained analytical approximations of the dispersion curves provide the solution of more complex problems of the stratified media wave dynamics. In particular, the constructed asymptotics of the dispersion relations further allow us to study the problem of studying the dynamics of IGW in the ocean by slowly varying and nonstationary parameters. In this case, the solution can be represented as a sum of wave train, the phase structure of which is determined by the analytical properties of the corresponding dispersion dependences. The phase functions (model integrals) of these asymptotic solutions are expressed in terms of various special functions: Fresnel integrals, Airy functions, Pearcey integrals [21, 22, 24]. The specific choice of phase functions (model integrals) is completely determined by the analytical properties of the dispersion relations.

\section{Conclusion.}

Numerical and analytical solutions that describe the main phase characteristics of IGW fields in a stratified ocean of finite depth, both for arbitrary and model distributions of the buoyancy frequency and background shear currents are studied in the work. For the analytical solution of the problem, a constant distribution of the buoyancy frequency and linear dependences of the background shear current on the depth were used. Model representations for the main hydrological characteristics (buoyancy frequency and background shear currents) make it possible to reduce the main spectral problem to a simpler one, and also to study this simplified spectral problem asymptotically. The obtained results show that asymptotic constructions, which use model representations of the buoyancy frequency and the distribution of background shear velocities, describe the spectral problem solutions with a good accuracy degree. The proposed asymptotic methods provide the analytical and qualitative investigation of wave dynamics, which is important for the operational analysis of field measurements of IGW in the ocean. Analytical expressions for dispersion curves can be used, in particular, for a qualitative interpretation of the observed wave phenomena in the ocean and for the development of efficient algorithms for detecting IGW using the radar methods.

\section{REFERENCES}

1. Miropol'sky, Yu.Z., 2001. Dynamics of Internal Gravity Waves in the Ocean. Dordrecht: Springer, 406 p. doi:10.1007/978-94-017-1325-2

2. Pedlosky, J., 2003. Waves in the Ocean and Atmosphere: Introduction to Wave Dynamics. Berlin: Springer, 264 p. doi:10.1007/978-3-662-05131-3

3. Fabrikant, A.L. and Stepanyants, Yu.A., 1998. Propagation of Waves in Shear Flows. Singapore: World Scientific Publishing, 304 p. https://doi.org/10.1142/2557 
4. Morozov, E.G., 2018. Oceanic Internal Tides: Observations, Analysis and Modeling: A Global View. Cham: Springer, 304 p. doi:10.1007/978-3-319-73159-9

5. Velarde, M.G., Tarakanov, R.Yu. and Marchenko, A.V., 2018. The Ocean in Motion: Circulation, Waves, Polar Oceanography. Cham: Springer, 625 p. doi:10.1007/978-3-31971934-4

6. Bulatov, V.V. and Vladimirov, Yu.V., 2019. A General Approach To Ocean Wave Dynamics Research: Modelling, Asymptotics, Measurements. Moscow: OntoPrint Publishers, 587 p.

7. Morozov, E.G., Tarakanov, R.Yu, Frey, D.I., Demidova, T.A. and Makarenko, N.I., 2018. Bottom Water Flows in the Tropical Fractures of the Northern Mid-Atlantic Ridge. Journal of Oceanography, 74(2), pp. 147-167. doi:10.1007/s10872-017-0445-X

8. Frey, D.I., Novigatsky, A.N., Kravchishina, M.D. and Morozov, E.G., 2017. Water Structure and Currents in the Bear Island Trough in July-August 2017. Russian Journal of Earth Sciences, 17(3), ES3003. doi:10.2205/2017ES000602

9. Khimchenko, E.E., Frey, D.I. and Morozov, E.G., 2020. Tidal Internal Waves in the Bransfield Strait, Antarctica. Russian Journal of Earth Sciences, 20(2), ES2006. doi:10.2205/2020ES000711

10. Miles, J.W., 1961. On the Stability of Heterogeneous Shear Flow. Journal of Fluid Mechanics, 10(4), pp. 496-508. https://doi.org/10.1017/S0022112061000305

11. Fraternale, F., Domenicale, L., Staffilani, G. and Tordella, D., 2018. Internal Waves in Sheared Flows: Lower Bound of the Vorticity Growth and Propagation Discontinuities in the Parameter Space. Physical Review E, 97(6), 063102. doi:10.1103/PhysRevE.97.063102

12. Gavril'eva, A.A., Gubarev, Yu.G. and Lebedev, M.P., 2019. The Miles Theorem and the First Boundary Value Problem for the Taylor-Goldstein Equation. Journal of Applied and Industrial Mathematics, 13(3), pp. 460-471. doi:10.1134/S1990478919030074

13. Khusnutdinova, K.R. and Zhang, X., 2016. Long Ring Waves in a Stratified Fluid over a Shear Flow. Journal of Fluid Mechanics, 794, pp.17-44. doi:10.1017/jfm.2016.147

14. Hirota, M. and Morrison, P.J., 2016. Stability Boundaries and Sufficient Stability Conditions for Stably Stratified, Monotonic Shear Flows. Physics Letters A, 380(21), pp. 1856-1860. doi:10.1016/j.physleta.2016.03.044

15. Churilov, S., 2018. On the Stability Analysis of Sharply Stratified Shear Flows. Ocean Dynamics, 68(7), pp. 867-884. doi:10.1007/s10236-018-1161-9

16. Tyugin, D., Kurkin, A., Pelinovsky, E. and Kurkina, O., 2012. Increase of Productivity of the Program Complex for Modeling of Internal Gravity Waves IGW Research with the Help of Intel ${ }^{\circledR}$ Parallel Studio XE 2013. Fundamentalnaya i Prikladnaya Gidrofizika, 5(3), pp. 89-95 (in Russian).

17. Bulatov, V.V. and Vladimirov, Yu.V., 2020. Dynamics of Internal Gravity Waves in the Ocean with Shear Flows. Russian Journal of Earth Sciences, 20(4), ES4004. doi:10.2205/2020ES000732

18. Bulatov, V. and Vladimirov, Yu., 2020. Analytical Approximations of Dispersion Relations for Internal Gravity Waves Equation with Shear Flows. Symmetry, 12(11), 1865. doi:10.3390/sym12111865

19. Borovikov, V.A. and Levchenko, E.S., 1987. The Green Function of the Internal Wave Equation in the Layer of Stratified Fluid with Average Shear Flows. Morskoy Gidrofizicheskiy Zhurnal, (1), pp. 24-31 (in Russian).

20. Bulatov, V.V. and Vladimirov, Yu.V, 2020. Internal Gravity Waves in the Ocean with Multidirectional Shear Flows. Izvestiya, Atmospheric and Oceanic Physics, 56(1), pp. 85-91. doi:10.1134/S0001433820010028

21. Fröman, N. and Fröman, P.O., 2002. Physical Problems Solved by the Phase-Integral Method. Cambridge: Cambridge University Press, 214 p. doi:10.1017/CBO9780511535086

22. Kravtsov, Yu.A. and Orlov, Yu.I., 1993. Caustics, Catastrophes and Wave Fields. Berlin: Springer, 228 p. doi:10.1007/978-3-642-97491-5 
23. Watson, G.N., 1995. A Treatise on the Theory of Bessel Functions. Cambridge: Cambridge University Press, 814 p.

24. Bulatov, V.V. and Vladimirov, Yu.V., 1990. The Propagation of Airy and Fresnel Internal Waves in a Horizontally Inhomogeneous Medium. Soviet Journal of Physical Oceanography, 1(6), pp. 501-506. doi:10.1007/BF02197007

About the authors:

Vitaly V. Bulatov, Leading Research Associate, Ishlinskiy Institute for Problems in Mechanics, Russian Academy of Sciences (101-1, Vernandskogo pr., Moscow, 119526, Russian Federation), Dr. Sci. (Phys.-Math.), Dr. Sci. (Economy), Professor, ResearcherID: M-5633-2015, Scopus Author ID: 35615830800, ORCID ID: 0000-0002-4390-4013, internalwave@mail.ru

Yury V. Vladimirov, Senior Research Associate, Ishlinskiy Institute for Problems in Mechanics, Russian Academy of Sciences (101-1, Vernandskogo pr., Moscow, 119526, Russian Federation), Ph. D. (Phys.-Math.), ResearcherID: M-5806-2015, Scopus Author ID: 7007066501, ORCID ID: 0000-0002-2139-5078, vladimyura@yandex.ru

Igor Yu. Vladimirov, Senior Research Associate, P.P. Shirshov Institute of Oceanology, Russian Academy of Sciences (36, Nakhimovsky Pr., Moscow, 117997, Russian Federation), Ph. D. (Phys.Math.), ResearcherID: AAY-4825-2020, Scopus Author ID: 54790327700, iyuvladimirov@rambler.ru

Contribution of the co-authors:

Vitaly V. Bulatov - conceptualization, problem formulation, investigation

Yury V. Vladimirov - methodology, analytical calculations

Igor Yu. Vladimirov - investigations, computer modelling

All the authors have read and approved the final manuscript. The authors declare that they have no conflict of interest. 